\title{
Generic Noninteger Order Controller for Time-Delay Systems
}

\author{
Sami Hafsi $\mathbb{D}^{1,2}{ }^{1,2}$ Sadem Ghrab, ${ }^{3}$ and Kaouther Laabidi ${ }^{2,3}$ \\ ${ }^{1}$ Université de Tunis El Manar, Ecole Nationale d'Ingénieurs de Tunis, Tunis, Tunisia \\ ${ }^{2}$ LR-11-ES20 Laboratoire d'Analyse, Conception et Commande des Systèmes, Tunis 1002, Tunisia \\ ${ }^{3}$ Université de Tunis, Ecole Nationale Supérieure d'Ingénieurs de Tunis, Tunis, Tunisia \\ Correspondence should be addressed to Sami Hafsi; samihafsi13@gmail.com
}

Received 27 March 2021; Revised 22 August 2021; Accepted 23 August 2021; Published 10 September 2021

Academic Editor: Omar Naifar

Copyright (c) 2021 Sami Hafsi et al. This is an open access article distributed under the Creative Commons Attribution License, which permits unrestricted use, distribution, and reproduction in any medium, provided the original work is properly cited.

\begin{abstract}
This paper focuses on the problem of fractional controller PI stabilization for a first-order time-delay systems. For this reason, we utilize the Hermite-Biehler and Pontryagin theorems to compute the complete set of the stabilizing $P I^{\lambda}$ parameters. The widespread industrial utilization of $P I D$ controllers and the potentiality of their noninteger order representation justify a timely interest in $P I^{\lambda}$ tuning techniques. Step responses are calculated through $\left(K_{p}, K_{i}, \operatorname{lambda}\right)$ parameters inside and outside stability region to prove the method efficiency.
\end{abstract}

\section{Introduction}

Time delay usually appears in many real-time engineering systems in the state, the measurements, or the control input $[1,2]$. Delays are involved in challenging areas of robotics and real-time systems [3], which are generally sources of instability. To eliminate this instability, researchers introduced several approaches of control among the conventional PID controller $[4,5]$. These controllers are the most used in industrial purposes due to their simplicity, easy designing method, and their setting methods [6]. Many tuning methods have been proposed in the literature to tune $P, P I$, and PID controllers [6].

After that, Podlubny has proposed a generalization of the classic $P I$ and $P I D$ controllers defined as $P I^{\lambda}$ and $P I^{\lambda} D^{\mu}$ where the order integrator $\lambda$ and the order differentiator $\mu$ assumed real noninteger values. Also, he proved that these types of fractional controllers are the best for dynamics systems control $[7,8]$. Using $P I^{\lambda} D^{\mu}$ allows one to choose besides the parameters of the classical PID $\left(K_{p}, K_{i}\right.$, and $\left.K_{d}\right)$ the orders of integration $\lambda$ and derivation $\mu$ [9]. In most cases, first-order plus dead time (FOPDT) can be used with success to represent the process dynamics of single-input single-output (SISO) systems [10, 11]. Fractional-order PID controllers have become an interest research topic in control engineering. It has been evolving along with the progress of the technology, and nowadays it is very often implemented in digital form rather than with pneumatic or electrical components. Also, fractional controllers are introduced as a solution to solve the problem of instability of time-delay systems, which give more performance for many types of systems [8]. Caponetto developed a new procedure to compute the parameters of fractional-order controller $P I^{\lambda} D^{\mu}$ designed to stabilize a first-order time delay systems [12]. Recently, Hafsi et al. has focused on the stabilization of first order with time-delay systems with a fractional controller of the form $P I^{\lambda}$ [13]. After that, an extension of this work has been developed in [14] for a fractional-order system with delay.

In this paper, we develop analytical characterization of the stabilizing set of fractional controllers for first-order systems with time delay. Based on an extension of the Hermite-Biehler theorem and the Pontryagin theorem, the necessary and sufficient conditions for the stability of SISO first-order systems with known time delay are derived. The algorithms for computing the stabilizing sets of $P I^{\lambda}$ controller are presented on the basis of these conditions. These algorithms simplify the tedious calculations of the intervals of the parameters $K_{p}$ and $K_{i}$ presented in papers $[13,14]$. 
The entire $\operatorname{set}\left(K_{p}, K_{i}, \lambda\right)$ is then found by sweeping the fractional order $\lambda$ over appropriate ranges.

This paper is structured as follows. Section 2 is an introductory step that presents the usual definitions of fractional calculus and also presents some advantages that characterize fractional control actions. Section 3 introduces the problem of the stabilization of time-delay systems and presents the theory of the analysis of systems with time-lags which are based on the Hermite-Biehler theorem. Section 4 is attempted to establish a fractional analysis theory for firstorder systems with time-delay systems controlled via a $P I^{\lambda}$ regulator. Finally, we present the results of the simulations in Section 5 .

\section{Fractional-Order Calculus: Preliminary Study}

Noninteger calculus can be considered as the generalization of classical calculus to fractional orders of integration or differentiation. The definitions of Riemann-Liouville (RL), Caputo, and Grûnwald-Letnikov are the most well-known definitions used in fractional calculus. According to Grunwald-Letnikov, fractional integration and differentiation orders are defined as follows [15]:

$$
{ }_{a} D_{t}^{\lambda} v(t)=\lim _{h \longrightarrow 0} h^{-\lambda} \sum_{j=0}^{[t / h]}(-1)^{j}(\lambda, j)^{T} v(t-j h),
$$

where $[t]$ represents the integer part of $t, h$ is the step size, $\left(\begin{array}{c}\lambda \\ j\end{array}\right)$ is a binomial coefficient, and the subscripts $a$ and $t$ denote the low limits related to the operation of fractional differentiation. Caputo's definition of the fractional derivative function $v(t)$ of order is defined as follows [8]:

$$
{ }_{a} D_{t}^{\lambda} v(t)=\frac{1}{\Gamma(\lambda-n)} \int_{a}^{t} \frac{\left(d^{n} v(\tau)\right) / d \tau^{n}}{(1-\tau)^{a-n+1}} d \tau, \quad-1<\lambda<n .
$$

The RL fractional integral or derivative of order is defined as follows [8]:

$$
{ }_{a} D_{t}^{\lambda} v(t)= \begin{cases}\frac{1}{\Gamma(-\lambda) !} \int_{a}^{t}(1-x)^{-\lambda-1} v(x) \mathrm{d} x, & \text { si } \lambda<0, \\ v(t), & \text { si } \lambda=0, \\ D^{n}\left[{ }_{a} D_{t}^{\lambda-n} v(x)\right], & \text { si } \lambda>0 .\end{cases}
$$

The Gamma function $\Gamma$ is defined by

$$
\Gamma(z)=\int_{0}^{\infty} e^{t} t^{z-1} \mathrm{~d} t
$$

\section{Fractional-Order Control Actions}

Noninteger order control is the use of fractional calculus to obtain a control system with fractional actions more efficiently than conventional actions. However, the system is modeled in a classical manner or fractional one. From a certain point of view, fractional-order control presented an evolution analogous to that of classical control, following two parallel paths depending on the starting point: the time domain or the frequency domain. In this section, we will look at control actions of type $K s^{\lambda}$ where $\lambda \in[-1,1]$.

$$
\begin{aligned}
& \lambda=-1: \text { integral action } \\
& \lambda=0: \text { proportional action } \\
& \lambda=1: \text { derivative action }
\end{aligned}
$$

The basic control actions traditionally considered will be special cases [8], in which in the closed-loop system shown in Figure 1, $r$ is the reference input, $u$ is the control signal, and $y$ is the output.

3.1. Complex Plane Analysis. In the complex plane, the roots of the system given in (1) are located according to the following equation [8]:

$$
1+K s^{\lambda} G(s)=0 .
$$

The magnitude and the phase are given by

$$
\begin{aligned}
K & =\frac{1}{\left|s^{\lambda}\right||G(s)|}, \\
\arg \left[s^{\lambda} G(s)\right] & =(2 k+1) \pi, \quad k=0, \pm 1, \pm 2, \ldots,
\end{aligned}
$$

or $s=|s| e^{j \theta}$, such that $\theta$ is the argument of $s$.

3.2. Fractional-Order Integral Action. Integral action slows down the system, eliminates steady-state error, and decreases relative stability [8]. In the frequency domain, the integral action increases the slopes of the amplitude curve by a coefficient $-20 \mathrm{~dB} / \mathrm{dec}$ and decrements the phase plots by a coefficient $\pi / 2 \mathrm{rad}$. In the complex plane, the integral action makes it possible to move the root locus of the system on the right half-plane [8]. On the other hand, the fractional integral control action is analyzed in the frequency domain as follows. The magnitude and the phase curves are, respectively, given by [8]

$$
20 \log \left[s^{\lambda} G(s)\right]_{s=j \omega}=20 \log |G(j \omega)|+20 \lambda \log (\omega) .
$$

The phase plot is given by

$$
\arg \left[s^{\lambda} G(s)\right]=\arg (G(s))+\lambda \frac{\pi}{2} .
$$

Accordingly, if we choose $\lambda$ in the interval $[-1 ; 0]$,

The magnitude curve is shifted with an interval value [-20 dB/dec $0 \mathrm{~dB} / \mathrm{dec}]$

The phase plot is delayed by an angle with respect to the interval $[-\pi / 2 \mathrm{rad}, 0]$

3.3. Fractional-Order Derivative Action. The derivative control action increases the relative stability but unfortunately it makes the system more sensitive to high frequency 


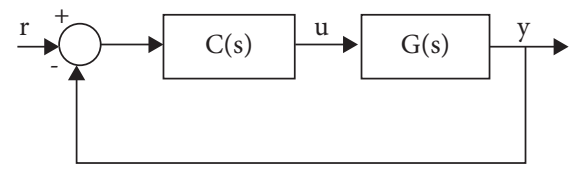

Figure 1: Block diagram of the unity feedback control system.

noise [8]. In the complex plane, it allows to move the locus of the roots of the system on the left half-plane. In the frequency domain, the derivative control action produces a constant phase advance of $\pi / 2 \mathrm{rad}$ and increases the slopes of the magnitude curves with a value equal to $20 \mathrm{~dB} / \mathrm{dec}$ [16]. By following a procedure similar to that of the integral action and from equations (7) and (8), we note that with the presence of the fractional derivative action [8],

The magnitude curve is shifted with an interval value [0dB/dec; $20 \mathrm{~dB} / \mathrm{dec}]$

The phase plot is delayed by an angle with respect to the interval $[0 ; \pi / 2 \mathrm{rad}]$

\section{Problem Characterization}

Many industrial processes can be characterized by a first order with time-delay transfer function:

$$
G(s)=\frac{K}{T s+1} e^{-L s}
$$

where $K$ represents the steady-state gain, $L$ represents the time delay, and $T$ represents the time constant for the plant. These three parameters are supposed to be positive. In this part, we consider the problem of stabilizing the first-order plus time-delay system with a noninteger regulator $P I^{\lambda}$.

$$
C(s)=K_{p}+\frac{K_{i}}{s^{\lambda}}
$$

The theory of fractional analysis, which is to be developed in this part, was based on the Hermite-Biehler theorem and was intended for first-order time-delay systems. Generally, this kind of system is characterized by the following relation:

$$
H(s)=b(s)+e^{-L_{1} s} m_{1}(s)+e^{-L_{2} s} m_{2}(s)+\cdots+e^{-L_{n} s} m_{n}(s),
$$

where $b(s)$ and $m_{j}(s)$ for $j=1,2, \ldots n$ are polynomials with real coefficients and $L_{1}, L_{2}, \ldots, L_{n}$ represent the delays. This type of equations, including $s$ and $e^{s}$ as variables, is called a quasi-polynomial and is used to characterize the closed-loop system when the transfer function involves time delay. This analysis is based on a new version of the Hermite-Biehler theorem for Hurwitz quasi-polynomials while respecting the following assumptions:

$$
\begin{aligned}
& A_{1}: 0<L_{1}<L_{2}<\ldots<L_{n}, \\
& A_{2}: \operatorname{deg}[b(s)]=m, \text { et } \operatorname{deg}\left[m_{j}(s)\right]<m .
\end{aligned}
$$

4.1. Hermite-Biehler Theorem. $\chi(j \omega)=\chi_{r}(\omega)+j \chi_{i}(\omega)$ where $\chi_{i}(\omega)$ and $\chi_{r}(\omega)$ are, respectively, the imaginary and the real part of the quasi-polynomial $\chi(j \omega)[17,18]$. Within $A 1$ and $A 2, \chi(j \omega)$ is stable if and only if

(1) $\chi_{r}(\omega)$ and $\chi_{i}(\omega)$ have only simple real roots, and these are interlaced

(2) $\left(\left(d \chi_{i}(\omega)\right) / \omega^{*}\right) \chi_{r}(\omega)-\left(\left(d \chi_{r}(\omega)\right) / \omega^{*}\right) \chi_{i}(\omega)>0$, for some $\omega \in]-\infty ;+\infty[\diamond$

4.2. Pontryagin Theorem. Let $M$ and $N$ indicate the highest powers of $s$ and $e^{s}$, respectively, in $\chi(j \omega)[17,18] . \varsigma$ is a suitable constant such that the coefficients of terms of highest degree in $\chi_{r}(\omega)$ and $\chi_{i}(\omega)$ do not vanish at $\omega=\varsigma$. As well, it is necessary and sufficient that in the intervals

$$
-2 l \pi+\varsigma \leq \omega \leq 2 l \pi+\varsigma \quad l=1,2,3, \ldots,
$$

the equations $\chi_{r}(\omega)=0$ and $\chi_{i}(\omega)=0$ have exactly $4 l N+M$ roots, starting with sufficiently large $l$.

\section{Stability Region for PI Fractional Controller}

According to the Hermite-Biehler theorem, the first condition ensures that the real and imaginary parts of the characteristic equation have only simple real roots and these are interlaced. The first part of this theorem is checked by the Pontryagin theorem.

The characteristic equation of the closed-loop system in Figure 1 is as follows:

$$
H(s)=\left(K K_{i}+K K_{p} s^{\lambda}\right) e^{-L s}+(1+T s) s^{\lambda} .
$$

Consider that the system to be controlled in this section is the first-order time-delay system presented by expression (9). Thus, our objective is to study the stability of the system, so we must analytically calculate all the parameters of the controller $K_{p}, K_{i}$, and $\lambda$ of the transfer function of the noninteger order controller given by expression (10). The real and imaginary parts of the characteristic relation become

$$
\begin{aligned}
H_{r}^{*}(\omega)= & K K_{i}+\left(K K_{p}+\cos (\omega)-\frac{T}{L} \omega \sin (\omega)\right)\left|\operatorname{Re}\left[(j)^{\lambda}\right]\right||\omega|^{\lambda}\left(\frac{1}{L}\right)^{\lambda} \\
& -\left(\sin (\omega)+\frac{T}{L} \omega \cos (\omega)\right)\left|\operatorname{Im}\left[(j)^{\lambda}\right]\right||\omega|^{\lambda}\left(\frac{1}{L}\right)^{\lambda} \operatorname{sign}(\omega), \\
H_{i}^{*}(\omega)= & \left(K K_{p}+\cos (\omega)-\frac{T}{L} \omega \sin (\omega)\right)\left|\operatorname{Im}\left[(j)^{\lambda}\right]\right||\omega|^{\lambda}\left(\frac{1}{L}\right)^{\lambda} \\
& * \operatorname{sign}(\omega)-\left.\left(\sin (\omega)+\frac{T}{L} \omega \cos (\omega)\right)\left|\operatorname{Re}\left[(j)^{\lambda}\right]\right| \omega\right|^{\lambda}\left(\frac{1}{L}\right)^{\lambda} .
\end{aligned}
$$

5.1. $K_{p}$ Range and Pontryagin Condition. Clearly here, $H_{r}^{*}(\omega)$ is function on the two variables $K_{p}$ and $K_{i}$ while the imaginary part $H_{i}^{*}(\omega)$ depends only on the parameter $K_{p}$. 
For this reason, let us now consider the system where the parameters are fixed like $L=4, K=1$, and $T=2$ and the fractional integral action $\lambda=0.5$. To ensure the stability of the closed-loop system, we first implement the Pontryagin theorem to determine the proportional gain $K_{p}$ which ensures the real roots of the imaginary part $H_{i}^{*}(\omega)$. This step allows us to define the best value of $\varsigma$ which gives the largest range of parameter $K_{p}$ for $-2 l \pi+\varsigma \leq \omega \leq 2 l \pi+\varsigma, l=1,2$, $3, \ldots$. This theoretical development of these results is quite involved in the following algorithm.

Here, $N_{r}$ represents the number of roots for $H_{i}^{*}(\omega)$ and $K_{p} 1$ and $K_{p} 2$ represent the first interval limits of $K_{p}$ arbitrary choose. The values of $K_{p}$ ensuring real roots for $H_{i}^{*}(\omega)$ are given by Algorithm 1 as shown in Figure 2. Note that the projection $\varsigma=0.8$ corresponds to the largest range for $K_{p}$.

From Figure 3, it is remarkable that, for some values inside this larger range of $-1<K_{p}<2.5$, the Pontryagin condition is respected. We can verify that for $K_{p}=-0.5$, $K_{p}=0$, and $K_{p}=1, H_{i}^{*}(\omega)$ has exactly five roots while for $K_{p}=3$, it has only one root. Therefore, out of this range, $H_{i}^{*}(\omega)$ does not have exactly five roots.
Since the value of $\varsigma$ is chosen, we calculate the range of the proportional gain $K_{p}$ for different values of $\lambda$ in the interval $[0.2 ; 1.8]$ as shown in Figure 4.

5.2. Solution of the Hermite-Beihler Conditions and Set of Stability Region. The next phase consists of calculating the intervals of $K_{p}$ and $K_{i}$ which satisfy the interlacing condition between the roots of the imaginary and real parts. From there, we remind that $K_{p}$ and $K_{i}$ affect the real part $H_{r}^{*}(\omega)$. However, it is enough to ensure that the interlacing property is respected between the roots of the real and imaginary parts of the characteristic equation, which also means that the real part has only simple real roots.

In fact, the real part of the characteristic equation can be written as follows:

$$
H_{r}^{*}(\omega)=K\left[K_{i}+K_{p} p(\omega)+q(\omega)\right]
$$

where

$$
p(\omega)=\left.\left|\operatorname{Re}\left\{(j)^{\lambda}\right\}\right| \omega\right|^{\lambda}\left(\frac{1}{L}\right)^{\lambda} \mid
$$

$$
q(\omega)=\frac{|\omega|^{\lambda}(1 / L)^{\lambda}}{K}\left[\operatorname{Re}\left\{(j)^{\lambda}\right\}\left(\cos (\omega)-\frac{T}{L} \omega \sin (\omega)\right)-\operatorname{Im}\left\{(j)^{\lambda}\right\}\left(\sin (\omega)+\frac{T}{L} \omega \cos (\omega) \operatorname{sign}(\omega)\right)\right]
$$

$$
a_{j}=(-1)^{j} p\left(\omega_{j}\right) K_{p}+(-1)^{j} q\left(\omega_{j}\right) \text {. }
$$

From equations (22)-(24), it is possible to develop the system of inequalities as follows:

$$
\left\{\begin{array} { l } 
{ H _ { r } ^ { * } ( 0 ) > 0 } \\
{ H _ { r } ^ { * } ( \omega _ { 1 } ) < 0 } \\
{ H _ { r } ^ { * } ( \omega _ { 2 } ) > 0 } \\
{ H _ { r } ^ { * } ( \omega _ { 3 } ) < 0 } \\
{ H _ { r } ^ { * } ( \omega _ { 4 } ) > 0 }
\end{array} \longrightarrow \left\{\begin{array}{l}
K_{i}>0 \\
K_{i}<a_{1} \\
K_{i}>a_{2} \\
K_{i}<a_{3} \\
K_{i}>a_{4}
\end{array} .\right.\right.
$$

Interlacing the roots of $H_{r}^{*}\left(\omega_{j}\right)$ and $H_{i}^{*}\left(\omega_{j}\right)$ is equivalent to $H_{r}^{*}(0)>0$ since $K_{i}>0, \quad H_{r}^{*}\left(\omega_{1}\right)<0, \quad H_{r}^{*}\left(\omega_{2}\right)>0$, $H_{r}^{*}\left(\omega_{3}\right)<0$, and so on.

Now, let us explore the conditions that allow us to verify whether the roots of $H_{r}^{*}\left(\omega_{j}\right)$ and $H_{i}^{*}\left(\omega_{j}\right)$ are interlaced. In fact, we compute the values of the real part $H_{r}^{*}\left(\omega_{j}\right)$ in the zeros of the imaginary part $H_{i}^{*}\left(\omega_{j}\right)$. Therefore, the interlacing property ensures that

$$
H_{r}^{*}(0)>0 \longrightarrow K K_{i}>0,
$$

which implies

$$
K_{i}>0
$$

for $\omega=\omega_{j} \neq 0$,

$$
\begin{gathered}
(-1)^{j} H_{r}^{*}\left(\omega_{j}\right)>0, \\
(-1)^{j} p\left(\omega_{j}\right) K_{p}+(-1)^{j} q\left(\omega_{j}\right)>(-1)^{j+1} K_{i} .
\end{gathered}
$$

Let $a_{j}$ be a complex parameter equal to
The approach developed previously is summarized in Algorithm 2.

We note that, $K_{p 1}$ and $K_{p 2}$ represent the terminals of the interval $K_{p}$ determined by the first algorithm. By applying Algorithm 2, we can find the stability region of the system $G(s)=1 /(2 s+1) e^{-4 s}$ as shown in Figure 5 . Note that for $K_{p}$ values outside this range, there is no stabilizing $P I^{\lambda=0.5}$ controller.

By sweeping over all $-1.4<K_{p}<1.5$, we can generate the set of values $\left(K_{p} ; K_{i} ; \lambda\right)$ for which the closed-loop system is stable. The region of global stability can then be visualized in a $3 \mathrm{D}$ plot as shown in Figure 6.

\section{Simulation Results}

In order to examine the accuracy of the stability region and the efficiency of our tuning method, step responses are calculated on either side of the stability region. Figures 7 and 8 
(1) Initialize step $=0.1 ; N_{r}=0 ; K_{p}=K_{p} 1$

(2) Count the roots of the equation $H_{i}^{*}(\omega)=0$ for $\omega \in[-2 \pi+\varsigma: 2 \pi+\varsigma]$

(3) If $N_{r}=4 l N+M$, then go to 4 ; else increment $K_{p}=K_{p}+$ step and go to 5 .

(4) Save $K_{p}$ and increment $K_{p}=K_{p}+$ step.

(5) If $K_{p}<K_{p} 2$, go to 2; else give Pontryagin interval.

Algorithm 1: $K_{p}$ vs. $\varsigma$ is calculated by considering the Pontryagin condition to the $P I^{\lambda}$ regulator.

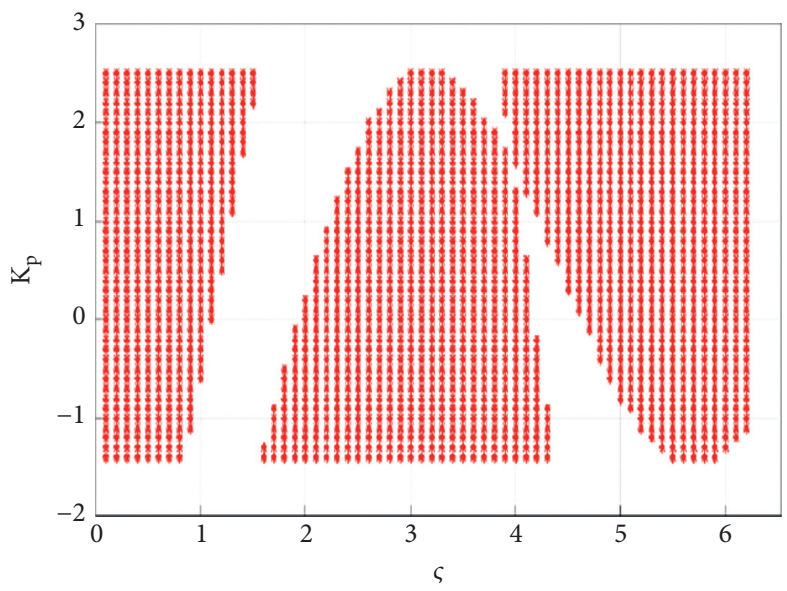

Figure 2: Evolution of $K_{p}$ for a $P I^{1 / 2}$ regulator.
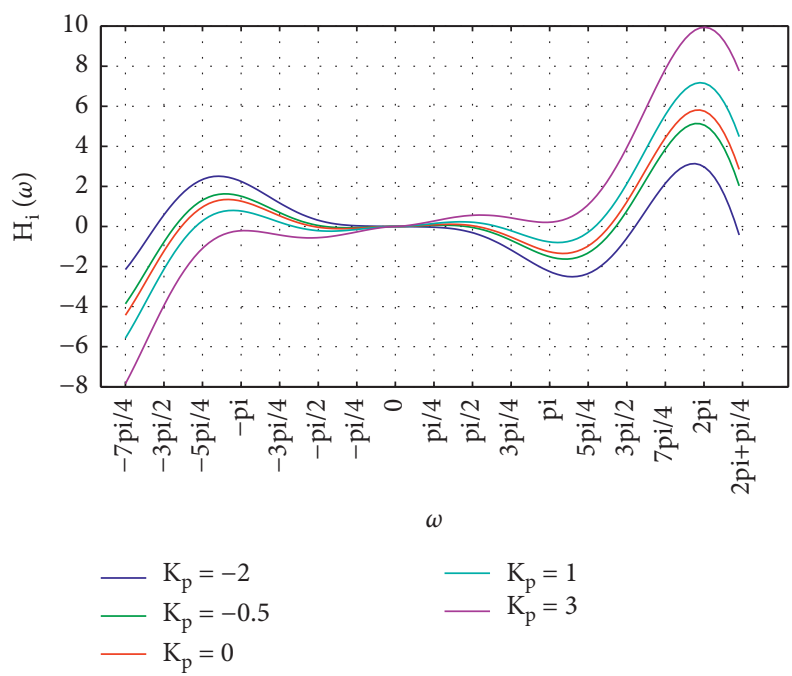

Figure 3: Trend of $H_{i}^{*}(\omega)$ for different values of $K_{i}$ for the $P I^{1 / 2}$ regulator.

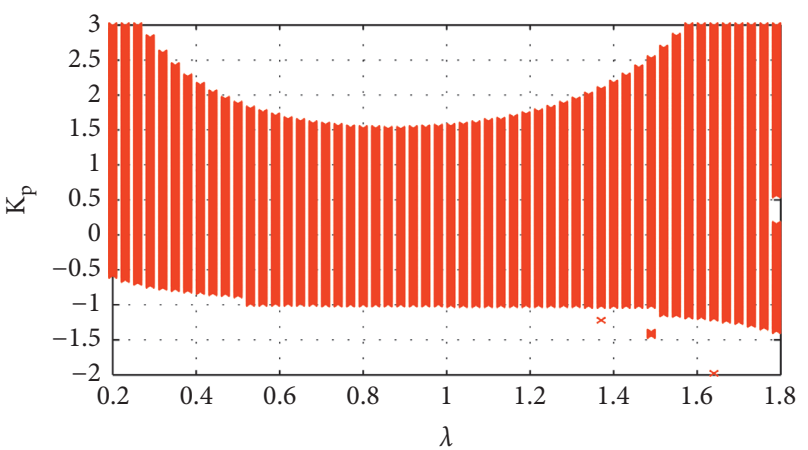

Figure $4: K_{p}$ vs. $\lambda$ as computed by applying the Pontryagin theorem to the $P I^{1 / 2}$ controller. 
(1) Initialize step $=0.1 ; N_{r}=0 ; K_{p}=K_{p} 1$.

(2) Compute the roots for $H_{r}^{*}(\omega)=0$.

(3) Determine the values of $K_{i}$.

(4) Increment $K_{p}=K_{p}+$ step; If $K_{p}<K_{p} 2$, then go to 2 ; else give the region $\left(K_{p} ; K_{i}\right)$.

Algorithm 2: Stability region for the system with fixed parameters.

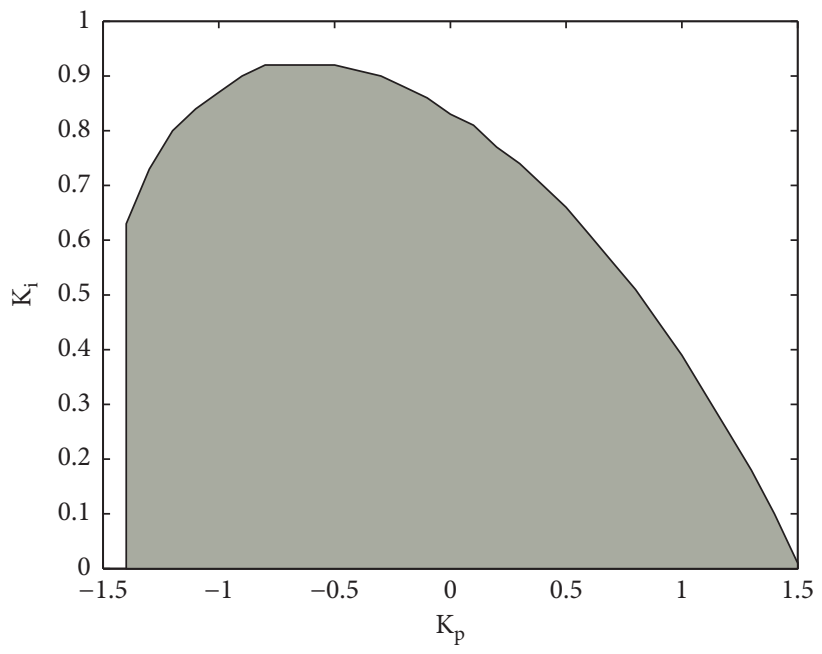

FIGURE 5: Stability region for $\lambda=0.5$.

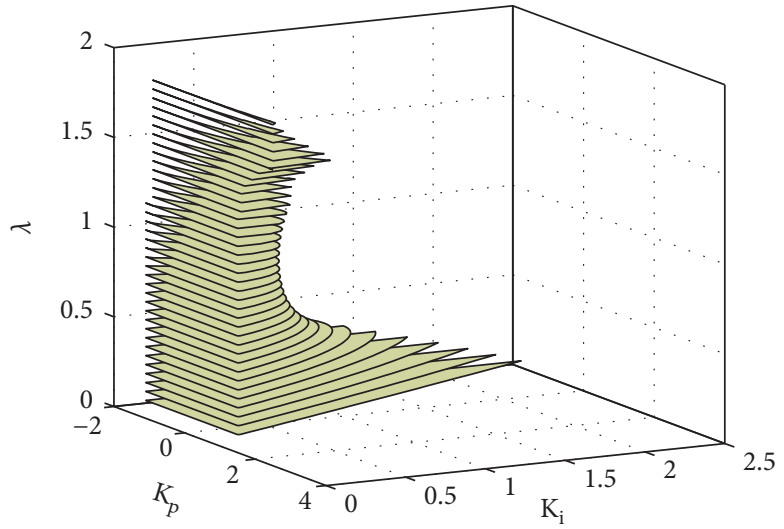

Figure 6: Global Stability region.

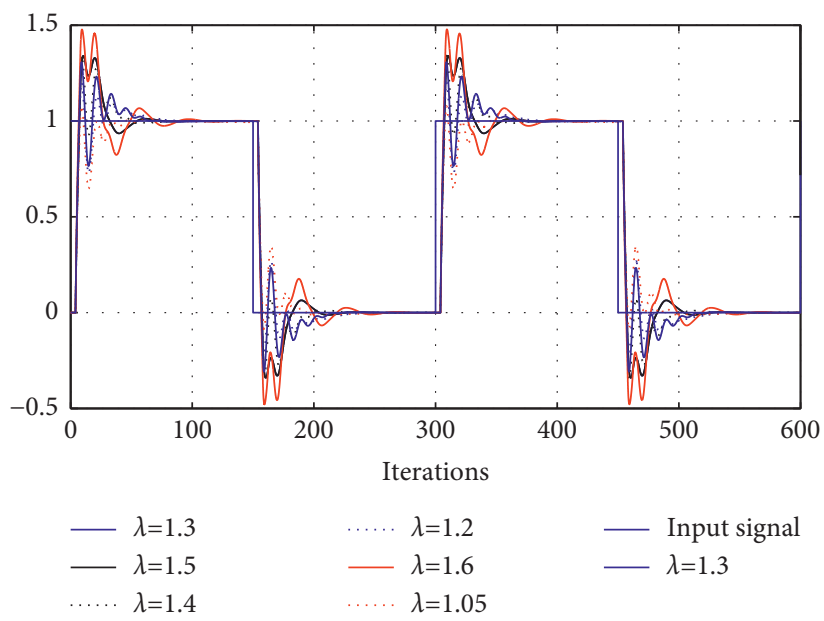

Figure 7: Responses to step for varying $\lambda>1$. 


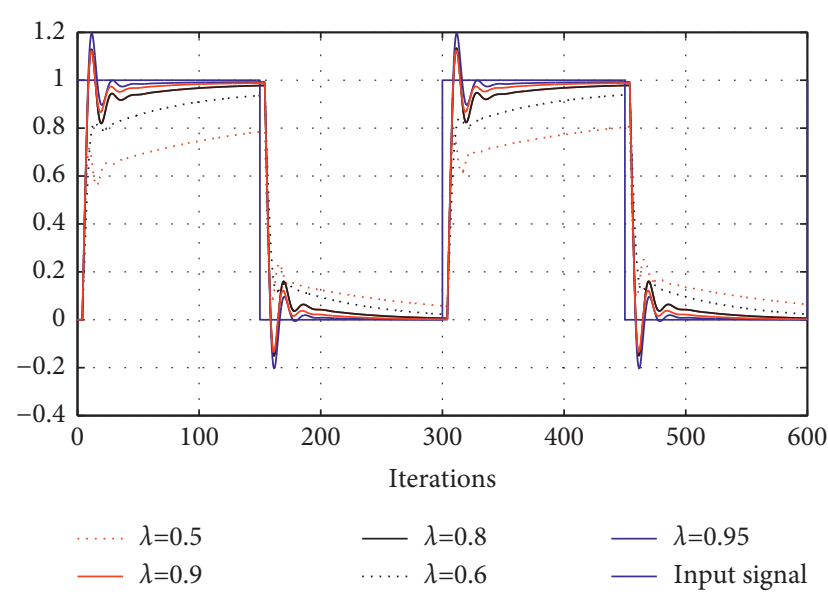

Figure 8: Responses to step for varying $\lambda<1$.

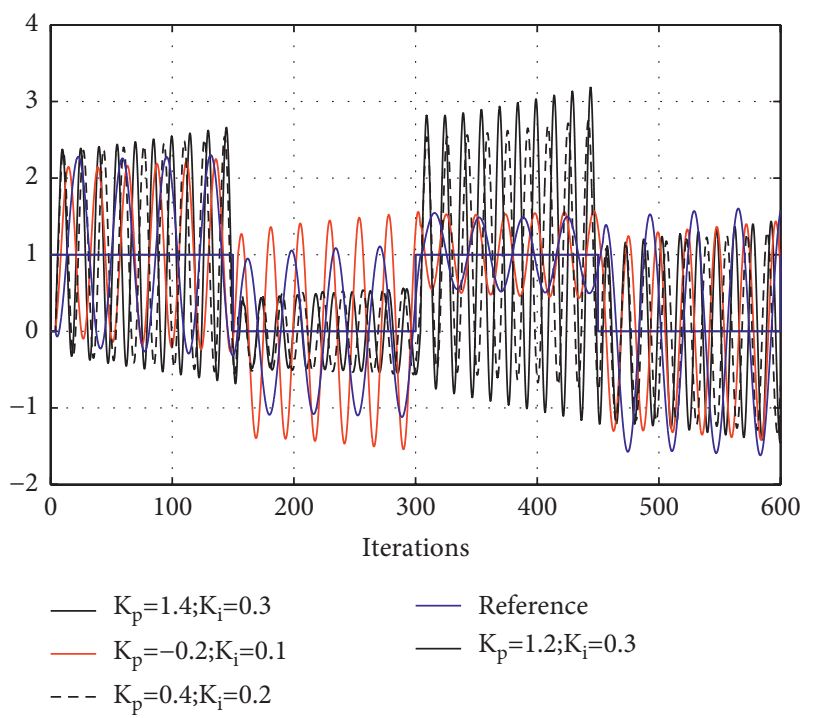

FIGURE 9: Responses to step for varying $\lambda=1.3$.

illustrate the calculation of the step responses with a value of the pair $\left(K_{p}, K_{i}\right)$ chosen within the interlimit of the stability region. On the other hand, Figure 9 shows the step responses calculated with a value of the pair $\left(K_{p}, K_{i}\right)$ choosing from the outer limit of the stability region. It is clear that the closedloop system has a convergent stable dynamic when the controller parameters choose within the internal limit of the stability region, and it has a divergent unstable dynamic when the controller parameters choose from the outer limit of the stability region.

We can also observe that for low $\lambda$ values, this gives high static error values; otherwise, that is, $\lambda$ greater than 1 , the static error is zero. It is interesting to note that the variation of the references is essential to evaluate the performances of the method developed in the presence of time delay.

\section{Conclusion}

In this work, we studied the stabilization problem of firstorder plus time-delay systems with fractional controller. The quasi-polynomials which model the delayed systems are characterized by an infinite number of roots which makes their study more complicated. In this case, a fractional analysis theory based on the Hermite-Biehler and Pontryagin theorems makes it possible to define a stability region for this kind of systems. The interval of $K_{p}$ was calculated by applying Pontryagin's theorem. According to these values, the roots of the imaginary part function are all simple and real. According to the interlacing property, for each value of $K_{p}$, there will be an interval of values of $K_{i}$ which represent the region of stability established for this fractional controller.

\section{Data Availability}

No data were used to support this study.

\section{Conflicts of Interest}

The authors declare that they have no conflicts of interest.

\section{References}

[1] J. Wu and L. Huang, "Global stabilization of linear systems subject to input saturation and time delays," Journal of the Franklin Institute, vol. 358, no. 1, pp. 633-649, 2021.

[2] N. Dushkov, I. Jordanov, and N. Vitanov, "Numerical modeling of dynamics of a population system with time delay," Mathematical Methods in the Applied Sciences, vol. 41, no. 18 , pp. 8377-8384, 2018.

[3] Q. Gao Hamid and R. Karimi, Stability, Control and Application of Time-Delay Systems, Elsevier, Physical Sciences and Engineering, Amsterdam, Netherlands, 1st edition, 2019.

[4] M. Zheng, T. Huang, and G. Zhang, "A new design method for PI-pd control of unstable fractional-order system with time delay," Complexity, vol. 2019, Article ID 3253497, 12 pages, 2019.

[5] C. Onat, "A new design method for PI-PD control of unstable processes with dead time," ISA Transactions, vol. 84, no. 1, pp. 69-81, 2019.

[6] G. J. Silva, A. Datta, and S. P. Bhattacharyya, PID Controllers for Time Delay Systems, Birkhäuser, Boston, MA, USA, 2005.

[7] I. Podlubny, "Fractional-order systems and $P I^{\lambda} D^{\mu}$ controllers," IEEE Transactions on Automatic Control, vol. 44, no. 1, pp. 208-214, 1999.

[8] C. A. Monje, Y. Q. Chen, B. M. Vinagre, X. Dingyu, and F. Vicente, Fractional-order Systems and Controls: Fundamentals and Applications (Advances in Industrial Control), Springer, Berlin, Germany, 2010.

[9] R. S. Barbosa, J. A. T. Machado, and I. M. Ferreira, "Tuning of PID controllers based on Bode?s ideal transfer function," Nonlinear Dynamics, vol. 38, no. 1-4, pp. 305-321, 2004.

[10] O. Waleed Abdulwahhab, "Design of a complex fractional Order PID controller for a first order plus time delay system," ISA Transactions, vol. 99, no. 1, pp. 154-158, 2018.

[11] L. Ribeiro, R. Costa Flesch, and J. Elias Normey-Rico, "Controlling industrial dead-time systems: when to use a PID or an advanced controller," ISA Transactions, vol. 99, no. 1, pp. 339-350, 2020.

[12] R. Caponetto, G. Dangola, L. Fortuna, and I. Petras, Fractional Order Systems Modeling and Control Applications, pp. 33-51, World Scientific Publishing Company, Singapore, 1st edition, 2010. 
[13] S. Hafsi, K. Laabidi, and R. Farkh, "Synthesis of a fractional PI controller for a first order time delay system," Transactions of the Institute of Measurement and Control, vol. 35, no. 8, pp. 997-1007, 2013.

[14] S. Gharab, S. Hafsi, and K. Laabidi, "Synthesis of PI fractional controller for fractional systems with time delay," in Proceedings of 2017 4th International Conference on Control, Decision and Information Technologies (CoDIT'17), Barcelona, Spain, April 2017.

[15] J. Sabatier and C. Farges, "Misconceptions in using RiemannLiouville's and Caputo's definitions for the description and initialization of fractional partial differential equations," IFAC Papers Online, vol. 50, no. 1, pp. 8574-8579, 2017.

[16] B. M. Vinagre and C. A. Monje, "Fractional-order PID," in PID Control in the Third Millennium, R. Vilanova and A. Visioli, Eds., pp. 465-493, Olympia, London, UK, 1st edition, 2012.

[17] S. Hafsi, K. Laabidi, and R. Farkh, "A new tuning method for stabilization time delay systems using $P I^{\lambda} D^{\mu}$ controllers," Asian Journal of Control, vol. 17, no. 3, pp. 1-11, 2015.

[18] R. Caponetto, G. Dongola, L. Fortuna, and A. Gallo, "New results on the synthesis of FO-PID controllers," Communications in Nonlinear Science and Numerical Simulation, vol. 15, no. 4, pp. 997-1007, 2010. 